\title{
Osteocyte RANKL in bone homeostasis: a paradigm shift?
}

$\mathrm{R}$

eceptor activator of nuclear factor- $\kappa \mathrm{B}$ ligand (RANKL) regulates osteoclast function, and its expression by osteoblasts is postulated to link bone resorption and formation. However, two independent studies, led by Hiroshi Takayanagi of the Tokyo Medical and Dental University and Charles O'Brien of the University of Arkansas for Medical Sciences, now challenge this paradigm by providing evidence that osteocytes are a major source of RANKL, and are essential for bone remodeling-a process that is defective in many rheumatic diseases.

"Many different cell types express RANKL and it remained unclear which ones were providing the RANKL involved in osteoclast formation in vivo," states O'Brien. His group and Takayanagi's initially took different approaches to attempt to fill this gap in our knowledge; Takayanagi et al. purified osteoblast and osteocyte populations, analysis of which revealed that RANKL levels were considerably higher in the latter, and the isolated osteocytes were more effective at driving the differentation of osteoclast precursors in vitro.

This discovery led Takayanagi et al. to generate knockout ( $\mathrm{KO}$ ) mice, hence forth referred to as Dmp1-RANKL KOs, with the gene encoding RANKL deleted specifically in osteocytes. Meanwhile, after initially finding that deletion of RANKL from all mesenchymal cells resulted in developmental defects, O'Brien et al. created their own Dmp1-RANKL KOs. In addition, O’Brien's group deleted RANKL at various genetically defined stages of osteoblast or chondrocyte development. Using these knockout mice, they showed that hypertrophic-chondrocyte-derived RANKL was essential for osteoclastogenesis during bone development, whereas no effect was seen in Dmp1-RANKL KOs. Though interesting, this finding did not provide much insight into the role of RANKL in non-developmental bone remodeling processes that are probably more relevant to rheumatic disease.

Takayanagi et al. also reported normal bone development in Dmp1-RANKL KOs,

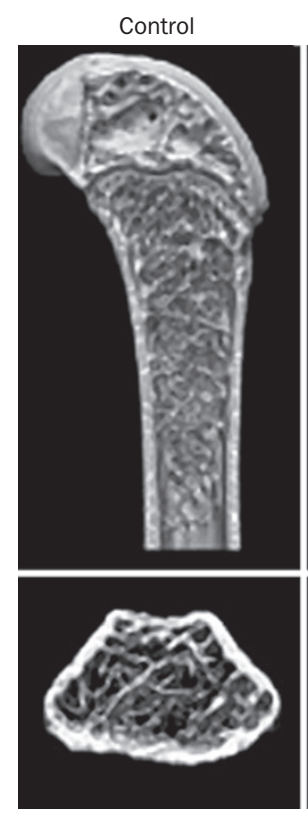

Dmp1-RANKL KO

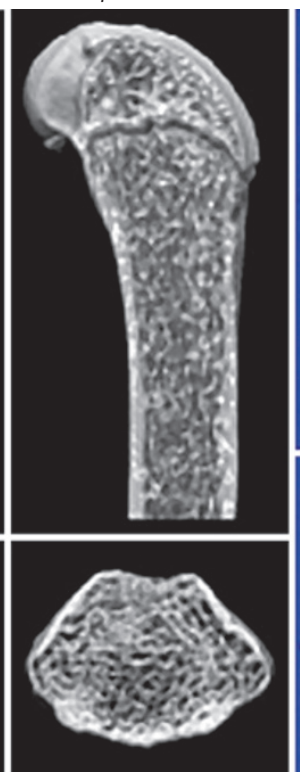

Control

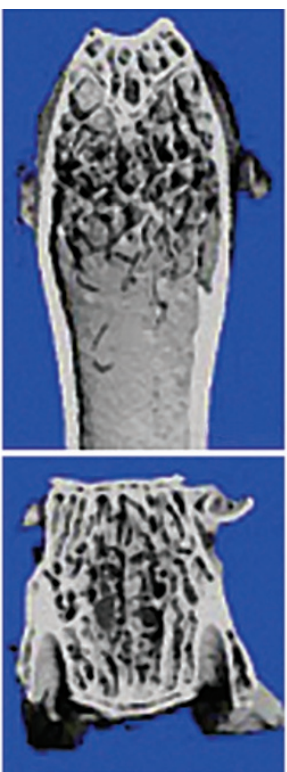

Dmp1-RANKL KO

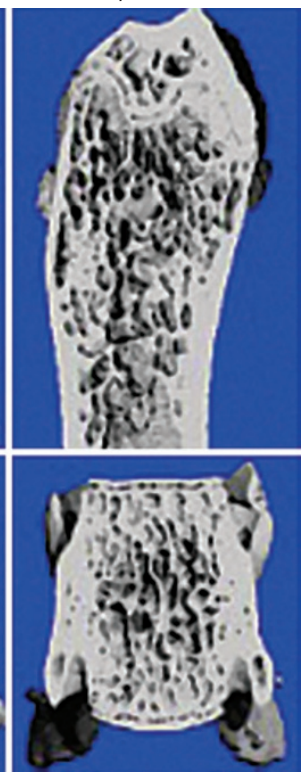

Micro-CT analysis of femurs longitudinal view (top left) and axial view (bottom left) of 12 week old mice from the Takayanagi study, and femurs (top right) or L4 vertebra (bottom right) of 6 month old mice from the O'Brien study. @ Nature Publishing Group.

but showed that osteocytes purified from these mice had reduced ability to support osteoclastogenesis in vitro, compared with those from control littermates. Interestingly, Takayanagi et al. found normal osteoclast numbers in Dmp1-RANKL KOs at birth, whereas both groups found a $>70 \%$ reduction postnatally compared with controls. "It was a big surprise that cells embedded within the bone matrix control osteoclast formation," claims O'Brien.

Both research groups went on to examine the role of osteocytes in bone homeostasis. Their combined data demonstrated that Dmp1-RANKL KO was associated with postnatal increases in bone mineral density (BMD) and volume compared with controls, and filling of the bone marrow cavity with bone. Additionally, markers of both bone formation and resorption were decreased in Dmp1-RANKL KO mice. These data suggest that, postnatally, "osteocytes are the major source of RANKL and thus regulate osteoclast formation and bone resorption," says Takayanagi.

O'Brien et al. also demonstrated a role for osteocyte RANKL in pathological bone remodeling using a model of unloading-induced bone loss; increases in RANKL mRNA and osteoclast numbers in bones of control mice were associated with changes in BMD, trabecular spacing and cortical thickness, but were not observed in Dmp1-RANKL KOs.

These findings are "...contrary to the preexisting dogma that RANKL is expressed by osteoblasts or bone marrow stromal cells, but consistent with the concept that osteocytes are mechanosensing cells that regulate other bone cells," states Takayanagi. "The concept put forward in our papers challenges a long-standing model...", adds O'Brien. "Now it will be important to determine whether RANKL produced by osteocytes is important in situations that lead to pathological bone loss, such as loss of sex steroids, aging, or inflammation," he continues.

\section{David Killock}

\footnotetext{
Original articles Xiong, J. et al. Matrix-embedded cells control osteoclast formation. Nat. Med. doi:10.1038/ nm.2448 | Nakashima,T. et al. Evidence for osteocyte regulation of bone homeostasis through RANKL expression. Nat. Med. doi:10.1038/nm.2452.
} 